\title{
Ready Posture for Rapid Reaction of Badminton Robot Arm
}

\author{
Satoshi Nishikawa \\ Department of Mechano-Informatics \\ University of Tokyo \\ 7-3-1 Bunkyo-ku, Tokyo, Japan \\ nisikawa@isi.imi.i.u-tokyo.ac.jp
}

\author{
Fumiya Iida \\ Department of Engineering \\ University of Cambridge \\ Trumpington Street, \\ Cambridge CB2 1PZ, United Kingdom \\ fi224@cam.ac.uk
}

\author{
Yasuo Kuniyoshi \\ Department of Mechano-Informatics \\ University of Tokyo \\ 7-3-1 Bunkyo-ku, Tokyo, Japan \\ kuniyosh@isi.imi.i.u-tokyo.ac.jp
}

\begin{abstract}
For immediate motion, taking a ready posture is a promising way because it can reduce enormous possible patterns of movement. For the selection of an appropriate ready posture, it is essential to know the structure of the task that includes the physical characteristics of a robot. We investigated our previous-built badminton robot arm to decide an appropriate ready posture as a case study. We first examined the kinematic characteristics to extract possible ready postures. In this analysis, we found that postures for hitting shuttles are limited to approximately one-tenth of all possible postures. Besides, these postures could be spatially divided into two clusters, one is for forehand shots, and the other is for backhand shots. Then, we calculated reaching time from various postures and confirmed that possible ready postures by kinematics analysis shortened averaged reaching time. We also found that there are more effective ready postures if considering the dynamics of the robot. These ready postures did not resemble those calculated by kinematics analysis.
\end{abstract}

Index Terms - Kinematics, Hydraulic/Pneumatic Actuators, Biologically-Inspired Robots

\section{INTRODUCTION}

Although rapid situational motion generation is essential for real-world robots, it has still remained a considerable challenge. Rapid situational motions can be regarded as motions of a robot required to rapidly react to different situations, as exemplified by ball sport such as tennis and badminton. Generally speaking, the generation of rapid situational motions is essential for many of these ball sports, but the technological solutions are also necessary beyond these entertainment platforms, more generally applicable for large variations of applications including rapid legged locomotion, dexterous manipulation, aggressive manoeuvres in UAVs, for example.

A popular approach to the generation of rapid situational motions is to employ high-speed sensors (e.g. vision, positional, and force sensors) for state recognition with high temporal resolutions, which is then used to rapidly compute motion trajectories, being executable by high-speed highprecision actuators. Dedicated structures are often adopted

${ }^{*}$ This work is supported by the Japan Society for the Promotion of Science through the KAKENHI Program under Grant JP18K18087, and the JSPS Invitational Fellowship for Research in Japan L19504.

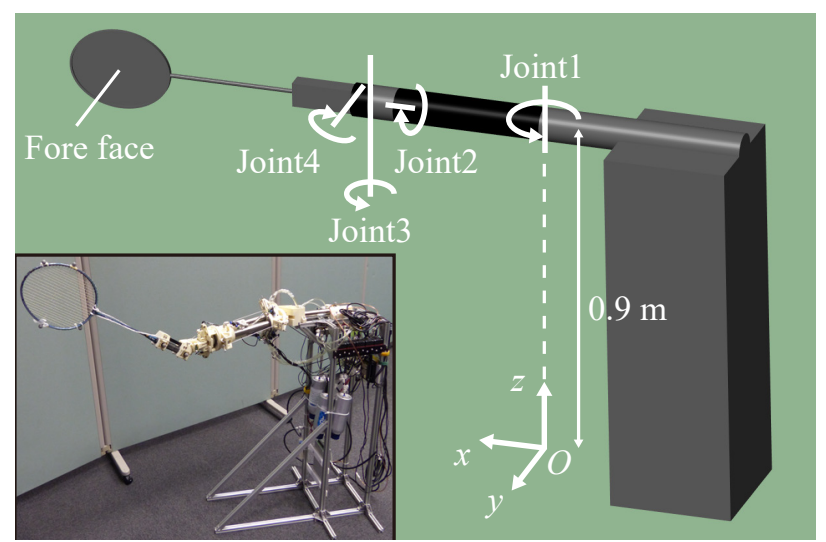

Fig. 1. Model of a badminton robot arm and its degrees of freedom. The robot model takes zero posture. Real robot [5] is shown in the left bottom frame.

to meet the demand for specific tasks. Such approaches have been shown previously in many ball sports robot platforms such as table tennis [1][2], baseball [3], or badminton [4]. While this approach is generally applicable to many different types of generating rapid situational motions, and often exceeds performances of humans, the solutions are usually high-cost and over-optimized for specific tasks being difficult to apply to different problems as human athletes playing well for many different types of games.

In contrast to these brute force technical solutions, humans tend to employ different strategies to achieve performances beyond their "hardware capabilities". For example, to generate motion immediately from many potential motion candidates, reducing possible motion patterns could be useful to reduce the computational cost for motion planning. Reduction of possible patterns is also effective for motor learning, such as for learning of local dynamics [6]. To reduce possible motion patterns, Imitation learning by human demonstration [7][8] is a promising approach. Some studies of table tennis robot adopted a virtual hitting plane to limit the possible hitting points [9][10] based on the virtual hitting point 
TABLE I

PHYSICAL PARAMETERS OF BADMINTON ROBOT ARM

\begin{tabular}{|c|c|c|c|c|}
\hline \multirow{2}{*}{ Joint } & \multicolumn{2}{|c|}{ Range of motion [rad] } & \multirow{2}{*}{$\begin{array}{l}\text { Length of the } \\
\text { next link [m] }\end{array}$} & \multirow{2}{*}{$\begin{array}{l}\text { Mass of the } \\
\text { next link }[\mathrm{kg}]\end{array}$} \\
\hline & $\min$ & $\max$ & & \\
\hline 1 & $-3 \pi / 4$ & $\pi / 4$ & 0.363 & 1.063 \\
\hline 2 & $-\pi / 2$ & $\pi / 2$ & 0.063 & 0.187 \\
\hline 3 & $-\pi / 2$ & $\pi / 2$ & 0.044 & 0.054 \\
\hline 4 & 0 & $\pi / 2$ & $0.584^{\mathrm{a}}$ & $0.254^{\mathrm{b}}$ \\
\hline
\end{tabular}

a The length to the center of the racket face

$\mathrm{b}$ The mass including a racket

hypothesis [11]. In addition, it is also important to consider the initial condition of posture, which we call "Ready Posture", with which the possible motion patterns can be easily generated without much computation in motion planning. In human sports, humans are known to take a specific posture before starting motions [12], which can be considered as an optimal ready posture to rapidly generate motions to react to different situations, even though it might deteriorate the performance of motion for more general cases. In general, finding such ready posture requires the knowledge about the task that includes the physical characteristics of a robot.

From this standpoint, this study investigates ready postures of a robot platform built for playing badminton (Fig. 1). In our previous study, we successfully demonstrated the basic capability of this robot reacting to a trajectory of badminton shuttle by selecting a motion from swinging motions defined in advance [5]. The initial postures of the robot was decided by a heuristic manner. In our other previous study, we examined the effect of ready posture for reaching using simple robot arm models with one or two degrees of freedom [13]. However, it dealt with general reaching with few constraints of the task.

In this study, in contrast, we focus on the ready posture of the specific platform, in order to react to different trajectories of shuttles, with minimum reaction time. As it becomes clearer in this paper, the ready posture plays a critical role in some cases, especially when the reaction time is highly restricted.

\section{METHOD}

To examine the effect of ready posture on the necessary time of hitting, we analyzed and simulated a badminton robot arm. First, we derived possible ready postures by kinematic analysis. Then, we calculated the required time for hitting from various initial postures. We used a model of a badminton robot arm that we developed before [5] (Fig. 1, Table I). Each joint of this robot is driven by a pneumatic cable cylinder to meet a demand for high acceleration and high speed. We used toolboxes of Matlab R2019a: Robotics System Toolbox for kinematic calculation, Simscape Multibody for dynamic simulation, and Deep Learning Toolbox

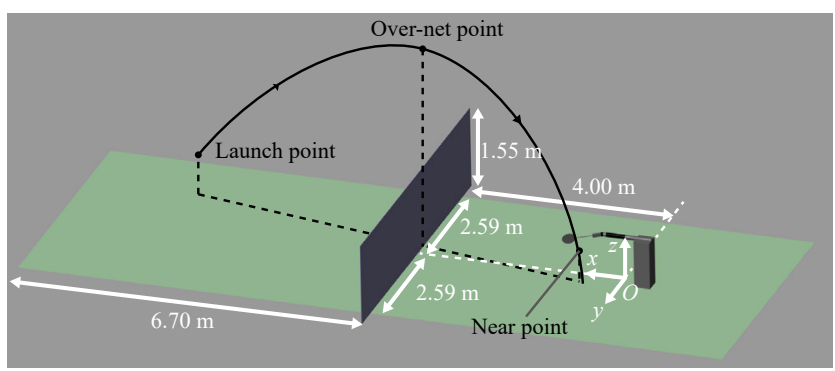

Fig. 2. The position of the robot in badminton court. The black curve is an example of a shuttle trajectory defined by three points.

for learning of neural networks.

In this study, we consider to use the robot platform shown in Fig. 1. The platform has a limited reachability because of the limitations of the hardware, but we consider hitting of shuttle within the reach of this platform. As shown in Fig. 2, even with these physical limitations, the platform is capable of reacting to many different situations, i.e. different trajectories of shuttles, determined by the initial conditions (positions and velocities) of shuttles. The following analyses in this paper were based on many randomly selected initial conditions of a shuttle, and consider how the robot can react to these variations because of the pertinent selection of ready postures.

\section{A. Calculation of postures by random sampling}

To understand the broad characteristics of robot kinematics for a badminton task, we extracted task-appropriate postures from random postures. First, we selected a set of random joint angles and calculated their positions and postures of a racket by solving forward kinematics. Next, we extracted a group of robot postures for hitting that meet the following condition.

$$
n_{\text {racket }, x} \cdot n_{\text {racket }, z}>0,\left|\frac{n_{\text {racket }, x}}{n_{\text {racket }, y}}\right|>\sqrt{3},
$$

where the normal vector of a racket is $\boldsymbol{n}_{\text {racket }}=$ $\left(n_{\text {racket }, x}, n_{\text {racket, } y}, n_{\text {racket }, z}\right)$. This condition means either side of a racket faces forward and upper direction. Then, we calculated the mean of these points and defined it as the ready posture kinematically determined by random sampling.

\section{B. Calculation of postures for random trajectories of a shuttle}

As a more task-specific case, we calculated hitting postures by random trajectories of a shuttle. First, we generated many trajectories of a shuttle as the following process. The robot model was on a badminton court, as shown in Fig. 2. Here, we used parabolic shuttle trajectories for simplification. Three points are necessary to determine the shape of the 
trajectory. We decided one point near the robot $q_{\text {near }}$ by random sampling in the following range (near point in Fig. 2).

$$
\begin{array}{r}
\left|q_{\text {near }}-q_{\text {base }}\right| \leq l_{\text {arm }}, \\
\left|q_{\text {near }, x y}\right| \leq l_{\text {forearm }}, \\
\left|q_{\text {near }, z}-q_{\text {base }, z}\right| \leq l_{\text {handracket }},
\end{array}
$$

where $q_{\text {base }}$ is the position of the first joint, $l_{\text {arm }}$ is total arm length, $l_{\text {forearm }}$ is forearm length (the summation length of first and second links), and $l_{\text {handracket }}$ is the length of hand and racket (the summation length of third and fourth links). We selected another points $q_{\text {launch }}$ as a launched position of a shuttle by selecting random points over the court of the opponent (launch point in Fig. 2). We set the range of height of these points as the reachable height of the robot, that is, $\left|q_{\text {launch }, z}-q_{\text {base }, z}\right| \leq l_{\text {handracket }}$. The horizontal projection of the third point is on the line created by connecting the horizontal projection of first and second points. We selected the third point $q_{\text {over-net }}$ above the net and randomly decided its height in the passing area. We set the upper end of the height to $5.0 \mathrm{~m}$ (over-net point in Fig. 2).

Next, we calculated hitting posture for each trajectory by calculating inverse kinematics for each point on the trajectory. We used generalizedInverseKinematics function as a solver of inverse kinematics. We chose the Broyden-FletcherGoldfarb-Shanno (BFGS) gradient projection algorithm. For calculation, we set the position of a racket, range of motion of each joint, and orientation of racket surface to the reverse direction of flying shuttle velocity with its angular tolerance as $0.1 \mathrm{rad}$ as constraints. If we got multiple points for hitting, we selected one hitting point randomly.

Then, we calculated the mean of these hitting points and defined it as the ready posture kinematically determined by random shuttle trajectories.

\section{Calculation of required time for hitting}

We calculated the required time for hitting from an initial posture as the procedure in the following paragraphs. Before the explanation of the procedure, we explain the setup of the dynamics simulation used in this study. We constructed a model of a badminton robot arm using physical parameters of the real robot [5]. For the calculation of actuator outputs from commands, we used the same pneumatic dynamics as described in Equations (5)-(8) of the previous study [5] because this robot has pneumatic cable cylinders as its actuators. The torque of $i$ th joint $\tau_{i}$ is calculated as;

$$
\tau_{i}=r_{\mathrm{MA}}\left(1-\mu_{\mathrm{w}}\right)\left(P_{i 1}-P_{i 2}\right)-\operatorname{sgn}\left(\omega_{i}\right) \tau_{\mathrm{c}},
$$

where $r_{\mathrm{MA}}$ is a moment arm, $\mu_{\mathrm{w}}$ is a coefficient of dynamic friction, $\tau_{\mathrm{c}}$ is a coefficient Coulomb viscous friction, $\omega_{i}$ is an angular velocity of $i$ th joint, and $P_{i j}$ is inner pressure of $j$ th pneumatic cylinder for $i$ th joint. $j=1$ means positive
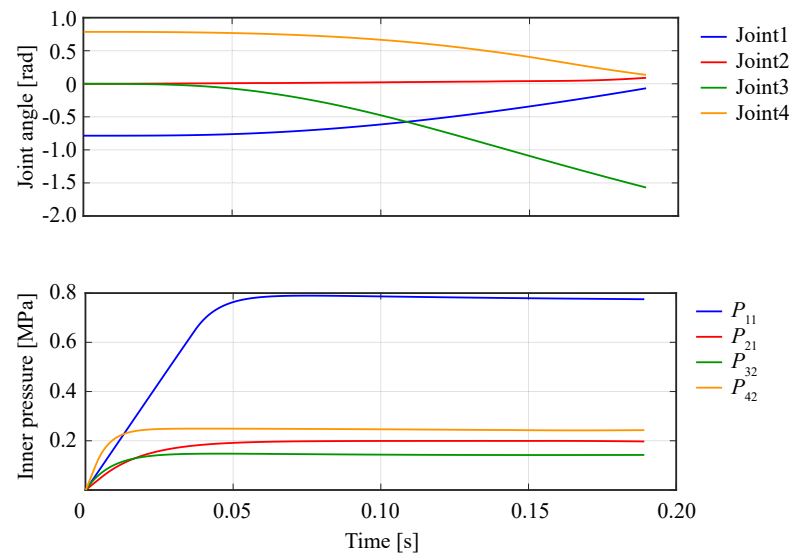

Fig. 3. An example of time series of joint angle and inner pressure of pneumatic cylinders during a swing. $P_{i j}$ is inner pressure of $j$ th pneumatic cylinder for $i$ th joint. $b=1$ means positive direction, and $b=2$ means negative direction. In this case, $P_{11}^{\text {target }}=0.80[\mathrm{MPa}]$, $P_{21}^{\text {target }}=0.20[\mathrm{MPa}], P_{32}^{\text {target }}=0.15[\mathrm{MPa}], P_{42}^{\text {target }}=0.25[\mathrm{MPa}]$, $P_{12}^{\text {target }}=P_{22}^{\text {target }}=P_{31}^{\text {target }}=P_{41}^{\text {target }}=0$ in the whole time. Here, we denote the target pressure for $P_{i j}$ as $P_{i j}^{\mathrm{target}}$.

direction, and $j=2$ means negative direction. The source pneumatic pressure was $0.9 \mathrm{MPa}$. The solver for simulation was the eighth-order Dormand-Prince method (ode8), and the simulation timestep was $0.1 \mathrm{~ms}$.

First, we simulated full swings to various directions from random sampled initial postures (Fig. 3). As shown in the graph of inner pressure, the convergence to the target pressure delayed though we provided the target pressure as a step function. Full swings were generated by setting a target innerpressure of either chamber of the pneumatic cylinder for one of the joints to the maximum value $0.8 \mathrm{MPa}$ and setting target inner-pressures of either chamber of the pneumatic cylinder for other joints to randomized values. Other target inner pressures were set to zero. As randomized values, we used uniform randomization for the first joint. We used Gaussian randomization, whose standard deviation was $0.2 \mathrm{MPa}$ for other joints. We stopped the simulation when one of the joint reached to the joint limit. We collected data by 100,000 swings.

Next, we regressed the required time for reaching from the combination of initial posture and final posture. We used a feedforward neural network for regression. We set the number of hidden layers as two and the number of neurons in each layer as 64 and eight. Training data were extracted from data collected in the previous paragraph by setting timestep for final posture as $T / \sqrt{1+|a|}$, where $T$ is timestep of the end of the simulation, and $a$ is random value with a standard normal distribution. Repeating this process ten times, we prepared 1,000,000 training data. Besides, we added 100,000 static data (the same initial and final postures) to the training data. We learned network ten times and adopted the average 


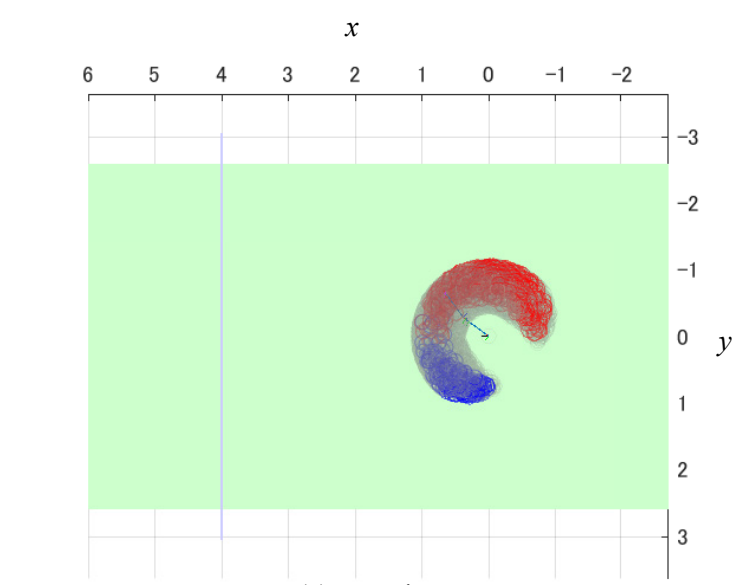

(a) Top view

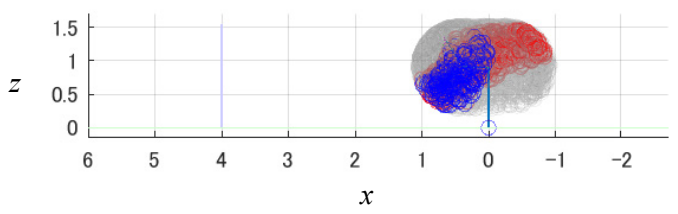

(b) Side view

Fig. 4. Reaching points by random postures. Red circles are for forehand shots. Blue circles are for backhand shots. Gray circles do not meet a requirement for racket postures.

of the output of them as an ensemble.

Then, we calculated averaged reaching time from ready posture calculated in the previous subsection to possible hitting postures using this network. Finally, we also calculated averaged reaching time from various initial postures to optimized ready posture, including dynamics property. We selected various initial postures as dividing the motion range of each joint among ten.

\section{RESULTS}

A. Ready posture kinematically determined by random sampling

Random sampling reaching points were located in a part of a torus shape (gray, red, and blue circles in Fig. 4). Among these points, points that are feasible for hitting were limited substantially (red or blue circles in Fig. 4). The number of points for forehand is 721 , and the number of points for backhand is 298 among 10000 points in total. To investigate the characteristics of the distribution of hittable postures in joint space, we drew histograms of joint angles (Fig. 5). The sum of red and blue histograms means the histogram for all hittable postures. As shown in the histograms, the distribution was not uniform. Therefore, the calculated ready posture was different from the posture with the middle range of motion
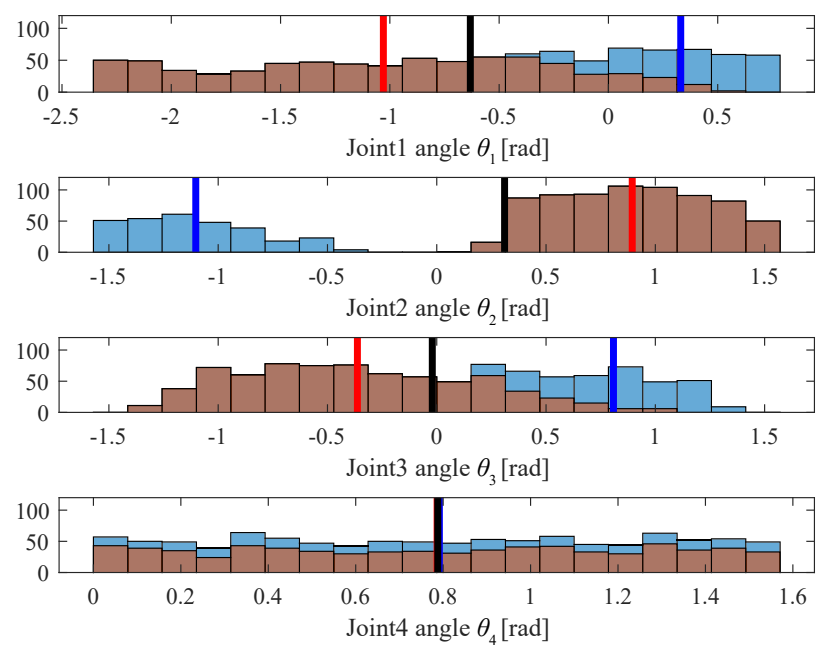

Fig. 5. Histogram of postures in random points reaching with restriction of racket postures. Red means points for forehand shots and blue means points for backhand shots. Vertical bold lines indicate the mean values for each shot. Black bold lines indicate the mean values of points for total shots.

(bold black line in Fig. 5). However, the deviation of ready posture from the middle posture was not large.

For more detailed analysis, we classified the postures to postures for forehand shots and those for backhand shots. The areas for forehand and backhand shots were nearly separated (red and blue circles in Fig. 4, respectively). The distribution in the joint one to three of each group biased to one side in the range of motion (red and blue histograms in Fig. 5). The distribution of postures for backhand shots had the opposite tendency against that for forehand shots. Thus, ready posture for each shot was different from that for whole shots (red and blue bold lines in Fig. 5). Therefore, if the necessary kind of shots could be identified beforehand, it is possible to take a more effective ready posture.

\section{B. Ready posture kinematically determined by random tra- jectories of a shuttle}

Hittable points for random trajectories of a flying shuttle appear to occupy a similar area to the random sampling describing in the previous subsection (Fig. 6). The trajectories for forehand and backhand shots were separated in horizontal projection (Fig. 6(a)), though sagittal trajectories were varied and mixed (Fig. 6(b)). This indicates that observing shuttle motion in horizontal projection is useful to decide the kind of shots. Distribution and ready postures also had a similar tendency to the random sampling case, except it had little more concentrated distribution (Fig. 7). This implies the constraint of racket face applied in the random sampling case captures the outline feature of this task, but a flying shuttle condition includes more tight constraints than the constraint described in (1). 


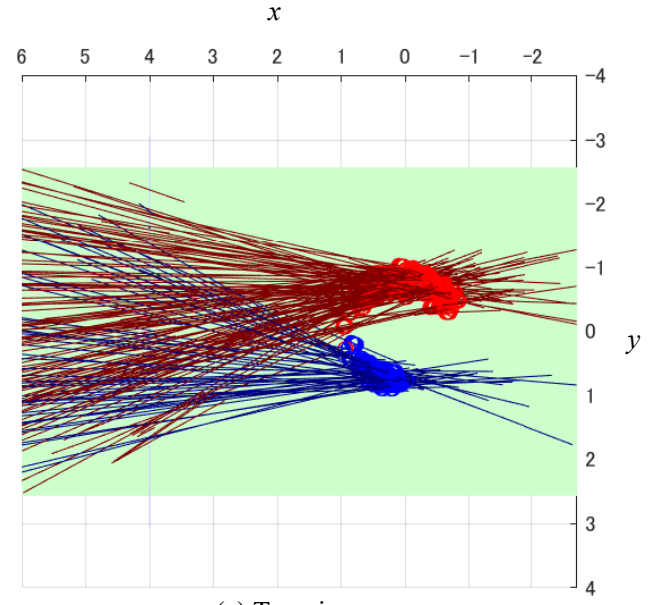

(a) Top view

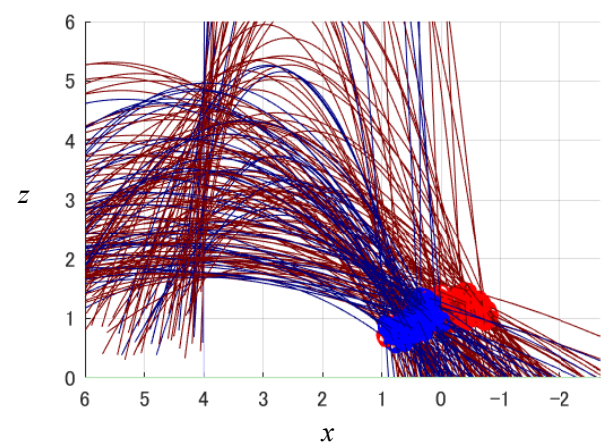

(b) Side view

Fig. 6. Random trajectories of a flying shuttle. Red circles indicate hittable points for forehand shots. Blue circles indicate hittable points for backhand shots.

\section{Required time for hitting from ready postures}

We compared averaged required time to reach from one of the three ready postures derived in the previous paragraph to the possible hitting postures. We considered three cases for possible hitting postures. One is with whole hitting postures, that is corresponding to have no information for trajectories. Another is with postures for forehand shots, that is corresponding to the case the robot can predict that it can hit a shuttle by forehand. The other is with postures for backhand shots, that is corresponding to the case the robot can predict that it can hit a shuttle by backhand. We also calculated averaged reaching time for all initial postures and the shortest averaged reaching time among searched initial postures. As a result, averaged required time with ready posture for whole hitting points was reduced from averaged reaching time from random initial postures (green and black in Fig. 8(a)). The same was going to with ready posture against the group of points for forehand shots (green and red in Fig. 8(b)). The reduction ratio was higher when using ready posture for backhand shots against the group
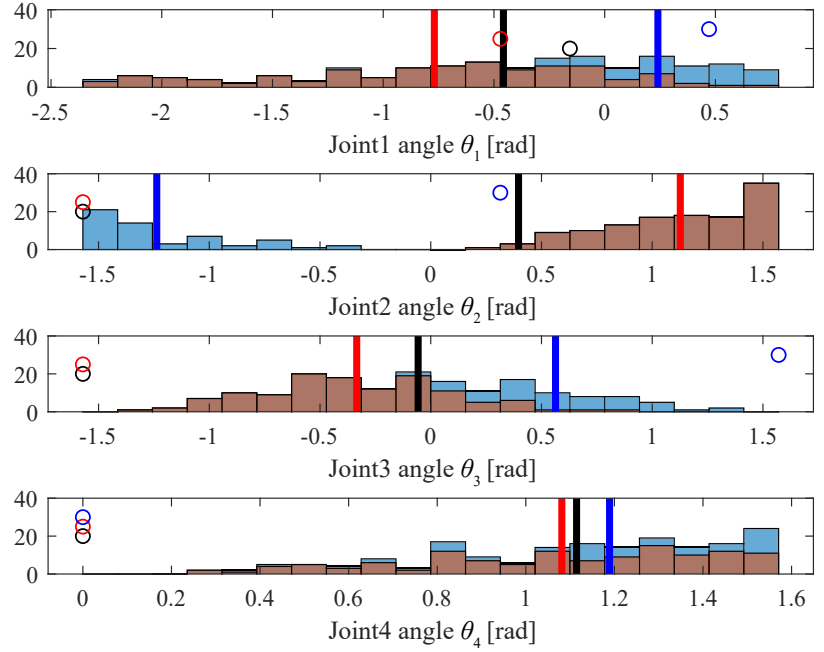

Fig. 7. Histogram of postures for hitting flying shuttles with random trajectories. Red means points for forehand shots and blue means points for backhand shots. Vertical bold lines indicate the mean values for each shot. Black bold lines indicate the mean values of points for total shots. Circles indicate the posture with the shortest reaching time for each shot.

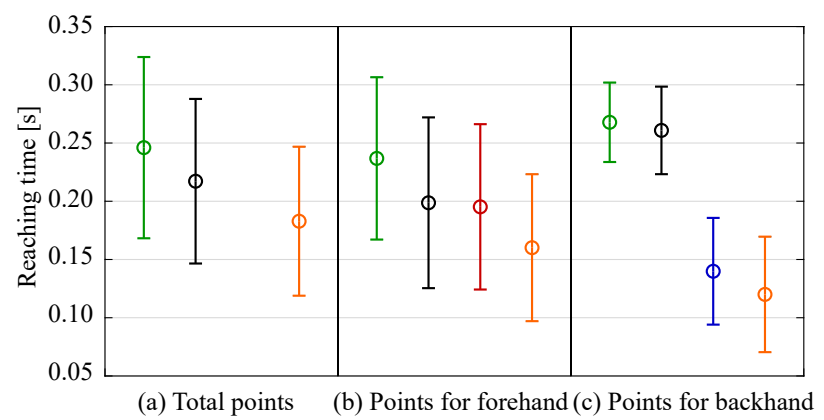

Fig. 8. Reaching time from initial posture to target posture in the flying shuttle condition. Green plots indicate the averaged reaching time for randomly-selected initial postures. Initial postures are the mean hittable posture (corresponding to bold black line in Fig. 7) in black, the mean hittable posture for forehand or backhand shots (corresponding to red or blue bold line in Fig.7) in red for forehand and blue for backhand, and the posture with shortest reaching time in each group of target postures in orange. In (a), (b), and (c), the group of target postures was set as whole hitting points, points for forehand hitting, and points for backhand hitting, respectively. Error bars indicate standard deviation.

of points for backhand shots (green and blue in Fig. 8(c)). However, the optimized postures had less reaching time than those with kinematic calculated ready postures (orange in Fig. 8). The optimized postures were largely different from those calculated by kinematics analysis (circles and bold lines in Fig. 7). The appearances of ready postures are shown in Fig. 9.

\section{DISCUSSION}

By the kinematics analysis, we found that effective postures of the shuttle hitting task were highly limited from 


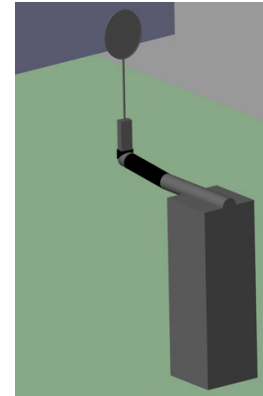

(a)

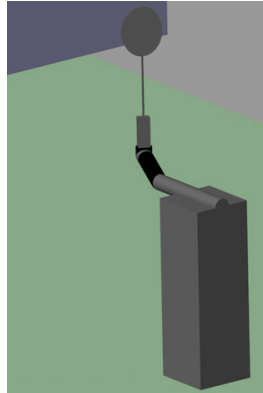

(b)

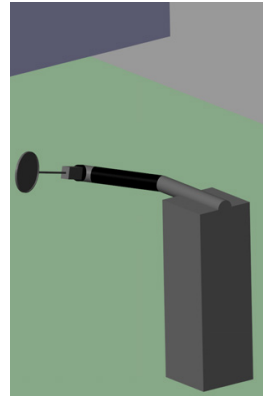

(c)
Fig. 9. Ready postures of minimal reaching time for hitting flying shuttles with random trajectories. (a) Ready posture for whole shots. (b) Ready posture for forehand shots. (c) Ready postures for backhand shots.

possible postures. This reduction of candidates suitable for a task could be useful not only for the decision of ready posture but also for the reduction of search space in motion learning or optimization. The reduction of search space is essential for the immediate reactive motion because the calculation time is limited in such motion.

The ready postures with the shortest reaching time were largely different from the averaged postures (circles and bold lines in Fig. 7). Although those of the first joint showed a relatively close tendency, those of other joints seem to have no relationship. This could be explained by the inertia for each joint. Because the first joint has large inertia, it is natural for the motion of the first joint to become a bottleneck of the reaching time. Besides, the inertia of the first joint is influenced by the posture of other joints. The postures for small inertia shorten the time to achieve the target angle of the first joint. Another factor for the difference is the interaction with joints by counteraction force. The direction of motion for one joint affects the motion speed of other joints. Due to the above factors, the relationship for reaching time by a joint angle was affected by the angles of other joints (Fig. 10).

Because the ready posture is used for the reduction of the search space, at least candidates must be obtained in advance. Thus, the calculation time for ready posture itself is not limited. Therefore, it is possible to use an exhaustive search considering dynamics.

\section{CONCLUSiOns}

We investigated the ready posture for a badminton robot arm by analyzing the kinematic structure of the robot and task constraints. We found that the number of possible postures for hitting was about one-tenth of all possible postures. When dividing hitting postures into postures for forehand shots and those for backhand shots, points of the center of racket face were separated, and angles of the first to the third joint have biased distribution. By calculating the reaching time from ready posture, we confirmed that the ready posture reduced

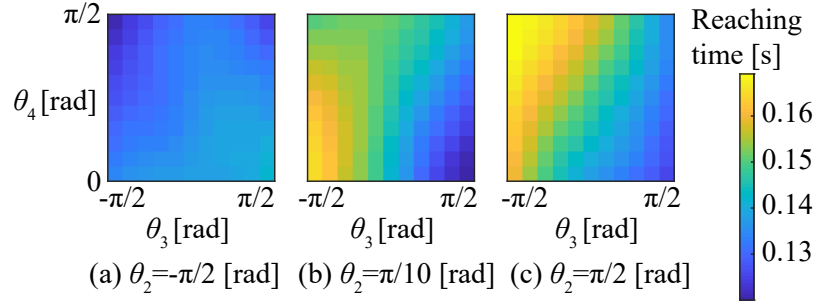

Fig. 10. Examples of reaching time by various initial postures for backhand shots. Here, $\theta_{1}=3 \pi / 20$ [rad].

reaching time, especially in the backhand case. Future work includes the improvement of the determination method of ready posture by coupling with motion generation, motor learning using ready posture, and the accomplishment of badminton task with a human by a robot.

\section{REFERENCES}

[1] H. Li, H. Wu, L. Lou, K. Kühnlenz, and O. Ravn, "Ping-pong robotics with high-speed vision system," in Proceedings of 12th International Conference on Control, Automation, Robotics and Vision (ICARCV), 2012, pp. 106-111.

[2] L. A. Silva, J. M. Sebastian, R. Saltaren, R. Aracil, and J. Sanpedro, "RoboTenis: Optimal design of a parallel robot with high performance," in Proceedings of IEEE/RSJ International Conference on Intelligent Robots and Systems (IROS), 2005, pp. 450-455.

[3] T. Senoo, A. Namiki, and M. Ishikawa, "Ball control in high-speed batting motion using hybrid trajectory generator," in Proceedings of IEEE International Conference on Robotics and Automation (ICRA), 2006, pp. 1762-1767.

[4] J. Stoev, S. Gillijns, A. Bartic, and W. Symens, "Badminton playing robot - a multidisciplinary test case in mechatronics," IFAC Proceedings Volumes, vol. 43, no. 18, pp. 725-731, 2010.

[5] S. Mori, K. Tanaka, S. Nishikawa, R. Niiyama, and Y. Kuniyoshi, "High-speed and lightweight humanoid robot arm for a skillful badminton robot," IEEE Robotics and Automation Letters, vol. 3, no. 3, pp. 1727-1734, 2018.

[6] V. Kumar, E. Todorov, and S. Levine, "Optimal control with learned local models: Application to dexterous manipulation," in Proceedings of IEEE International Conference on Robotics and Automation (ICRA), 2016, pp. 378-383.

[7] K. Mülling, J. Kober, O. Kroemer, and J. Peters, "Learning to select and generalize striking movements in robot table tennis," The International Journal of Robotics Research, vol. 32, no. 3, pp. 263-279, 2013.

[8] O. Koç and J. Peters, "Learning to serve: an experimental study for a new learning from demonstrations framework," IEEE Robotics and Automation Letters, vol. 4, no. 2, pp. 1784-1791, 2019.

[9] K. Mülling, J. Kober, and J. Peters, "A biomimetic approach to robot table tennis," Adaptive Behavior, vol. 19, no. 5, pp. 359-376, 2011.

[10] Z. Wang, A. Boularias, K. Mülling, B. Schölkopf, and J. Peters, "Anticipatory action selection for human-robot table tennis," Artificial Intelligence, vol. 247, pp. 399-414, 2017.

[11] M. Ramanantsoa, "Towards a stroke construction model," International Journal of Table Tennis Science, vol. 2, pp. 97-114, 1994.

[12] T. M. Johnson, L. E. Brown, J. W. Coburn, D. A. Judelson, A. V. Khamoui, T. T. Tran, and B. P. Uribe, "Effect of Four Different Starting Stances on Sprint Time in Collegiate Volleyball Players," Journal of strength and conditioning research, vol. 24, no. 10, pp. 2641-2646, 2010.

[13] K. Tanaka, S. Nishikawa, and Y. Kuniyoshi, "Effect of preliminary motions on agile motions," in Proceedings of 16th International Conference on Advanced Robotics (ICAR), 2013. 